\title{
Frequency of bla TEM, bla SHV, bla CTX-M, and qnrA Among Escherichia coli Isolated From Urinary Tract Infection
}

\author{
Shima Abdi ${ }^{1}$; Reza Ranjbar ${ }^{2}$; Mojdeh Hakemi Vala ${ }^{3, ;}$; Nematollah Jonaidi ${ }^{4}$; Ozra Baghery \\ Bejestany ${ }^{2}$; Fatemeh Baghery Bejestany ${ }^{1}$ \\ ${ }_{1}^{1}$ Department of Microbiology, Pharmaceutical Sciences Branch, Islamic Azad University, Tehran, IR Iran \\ ${ }^{2}$ Molecular Biology Research Center, Baqiyatallah University of Medical Sciences, Tehran, IR Iran \\ ${ }^{3}$ Department of Microbiology, Shahid Beheshti University of Medical Sciences, Tehran, IR Iran \\ ${ }^{4}$ Health Research Center, Baqiyatallah University of Medical Sciences, Tehran, IR Iran \\ *Corresponding author: Mojdeh Hakemi Vala, Department of Microbiology, Medical School, Shahid Beheshti University of Medical Sciences, Tehran, IR Iran. Tel: +98-2123872556, Fax: \\ +98-2122439964, E-mail:m.hakemi@sbmu.ac.ir
}

Received: March 2, 2014; Accepted: May 10, 2014

Background: Escherichia coli is the most important as well as the most common bacteria causing urinary tract infections (UTIs) and its resistance to common antibiotics is increasing. Extended-spectrum beta-lactamase (ESBL) producer E. coli strains can resist against the third-generation and fourth-generation cephalosporins.

Objectives: This study aimed to evaluate the resistance profile of $E$. coli isolated from patients with UTIs referred to Imam Khomeini and Baqiyatallah Hospitals, Tehran, Iran, through phenotypic and molecular methods.

Materials and Methods: During 2010-2011,180 urine samples of patients with UTIs from Imam Khomeini and Baqiyatallah Hospitals were collected. Based on the standard bacteriologic tests, E. coli isolates were identified. Resistance to common antibiotics was tested by the Kirby-Bauer method and reconfirmed by determining minimum inhibitory concentration (MIC) through microdilution method. Further phenotypic double-disk synergy test (DDST) was performed to screen the ESBL producer strains. Resistance genes related to ESBL and qnrA were evaluated by Polymerase chain Reaction(PCR).

Results: A total of 100 E. coli strains were examined by antibiogram and the rates of resistance to the tested antibiotics were as follows: $100 \%$ to penicillin and amoxicillin, $77 \%$ to amoxicillin-clavulanic acid, $72 \%$ to ceftazidime, $69 \%$ to cefotaxime, $47 \%$ to cefoxitin, $46 \%$ to ceftriaxone, $43 \%$ to cephalexin, $27 \%$ to aztreonam, $53 \%$ to nalidixic acid, $51 \%$ to ciprofloxacin, and $2 \%$ to imipenem. The MIC to ciprofloxacin, cefazolin, and ceftriaxonewere $\geq 0.249, \geq 0.508$, and $\geq 0.044$, respectively. Moreover, $20 \%$ of $E$. coli isolates were ESBL-producing isolates byDDST.The frequency of bla CTX-M, bla TEM, bla SHV, and qnrA genes was $87 \%, 82 \%, 65 \%$, and $39 \%$, respectively.

Conclusions: Considering the high prevalence of ESBL genes (bla CTX-M, 87\%; and bla TEM, 82\%), fluoroquinolones may be used as an alternative drug in treatment, although resistance to this family is increasing as well. As a result, this increasing trend should be prevented using appropriate guidelines for prescription.

Keywords:Escherichia coli; bla CTX-M; bla TEM; beta-Lactamases; bla SHV

\section{Background}

Escherichia coli is the most common bacterial agent causing urinary tract infections (UTIs). Cephalosporins, especially the third-generation ones, are used as a routine treatment for UTIs and fluoroquinolones are used increasingly as new alternative treatment of choice (1). During the recent years, these bacteria have undergone mutation and became resistant to many antibiotics by exchanging the resistant genes (2). One of the resistance mechanisms is producing enzymes like beta-lactamase and extended-spectrum beta-lactamases (ESBLs) that can hydrolyze the beta-lactam ring and inactivate these drugs that contain this structural element. Major reports of ESBL production are related to bla CTX-M, bla TEM and bla $S H V$ as plasmid genes, which can simultaneously carry other resistant genes (3-7). As mentioned before, the fluoroquinolones are another choice for treatment of betalactam-resistant bacteria $(8,9)$. Similarly, some plasmid genes like $q n r A$ are responsible for quinolone resistance.

\section{Objectives}

The aim of this study was to evaluate the resistance profile of E. coli, isolated from urine samples of the patients with UTIs, to routine antibiotics especially beta-lactams and ciprofloxacin. 


\section{Materials and Methods}

In this study, 180 urine samples were collected from patients with UTI referred to Imam Khomeini and Baqiyatallah Hospitals, Tehran, Iran, during September 2010 to March 2011. After special identification tests including EMB (eosin-methylene blue agar), TSI (Triple Sugar Iron agar), $\mathrm{SIM}\left(\mathrm{SH}_{2}\right.$, Indole and Motility), MR-VP (Methyl Red VogesProskauer Broth), and Citrate, 100 E. coli isolates were randomly selected for further investigation. Their resistance to common antibiotics, namely, imipenem, ceftriaxone, penicillin, amoxicillin, amoxicillin-clavulanic acid, ciprofloxacin, nalidixic acid, cefixime, cephalexin, cefotaxime, ceftazidime, and aztreonam discs (all from Himedia Company, India) was assessed according to the Clinical and Laboratory Standards Institute (CLSI) protocol and by the Kirby-Bauer method (10). In further step, minimal inhibitory concentration (MIC) was determined by microdilution method for ceftriaxone, cefazolin, and ciprofloxacin in accordance with CLSI protocol (10). Simultaneously, E. coli ATCC 25922 was used as the control. In the next step, ESBL production was assessed by double-disk synergy test (DDST) and using cefotaxime with and without clavulanic acid and ceftazidime with and without clavulanic acid discs. In this test, the antibiotic disks alone and with clavulanic acid were placed $25 \mathrm{~mm}$ apart from each other (10). More than or equal to $5 \mathrm{~mm}$ increase in the diameter of the growth inhibition zone of the antibiotic with clavulanic acid in comparison to the disk alone showed positive results for the ESBL production $(11,12)$. For molecular investigation, DNA extraction was done using DNA Extract Kit (Taif Ara, Iran) and consequently polymerase chain reac- tion (PCR) method was employed to assess the frequency of resistance genes including bla CTX-M, bla SHV, and bla TEM for ESBLs genotyping and qnrA gene for ciprofloxacin resistance study $(13,14)$. More details about the sequence of used primers and PCR programming are shown in Tables 1 and 2 . The PCR products were analyzed after gel electrophoresis, stained by ethidium bromide, and exposed to the UV radiation. During PCR, ESBL-positive Klebsiella pneumonia ATCC 700603 was used as the positive control.

\section{Results}

According to the identification tests, 100 confirmed E. coli isolates were selected for the experiment; $79 \%$ of the samples were collected from adults (females, 51\%; and males, $28 \%$ ) and $21 \%$ from children. The frequency of the samples based on the hospital wards was as follows: gynecology, $36 \%$; urology, $15 \%$; neurology, $14 \%$; miscellaneous wards, $10 \%$; and outpatient clinics, $25 \%$. According to the antibiogram test, the rates of resistance of the bacteria to antibiotics was as follows: amoxicillin and penicillin, $100 \%$; ceftazidime, $72 \%$; cefotaxime, $69 \%$; nalidixic acid, $53 \%$; ciprofloxacin, $51 \%$; cefoxitin, 47\%; cephalexin, 43\%; aztreonam, 27\%; and imipenem, $2 \%$ (Figure 1). In microdilution test, the MIC of resistant strains to ceftriaxone, cefazolin, and ciprofloxacin was $\geq 0.044, \geq 0.508$, and $\geq 0.249$, respectively.ByDDSTtest, $20 \%$ of the E. coli isolates showed $\geq 5 \mathrm{~mm}$ increase in the diameter of the growth inhibition zone around the clavulanic acid disks and were marked as ESBL-positive isolates (Figure 2). In the PCR test, after preparing the required conditions, the frequency of bla CTX-M, bla TEM, bla SHV, and qnrA genes was $87 \%, 82 \%, 65 \%$, and $40 \%$, respectively (Figures 3 and 4 ).

Table 1. The Sequence of Primers Used in This Study ${ }^{a}$

\begin{tabular}{|c|c|}
\hline Primers & Sequences \\
\hline bla TEM & F: 5' - ATAAAATTCTTGAAGACGAAA - 3'R: 5' - GACAGTTACCAATGCTTAATCA - 3' \\
\hline bla CTX-M & F: 5' - ACGCTGTTGTTAGGAAGTG -3'R: 5' - TTGAGGCTGGGTGAAGT - 3' \\
\hline bla SHV & F: 5' - GGGTTATTCTTATTTGTCGC-3'R: 5' - TTAGCGTTGCCAGTGCTC - 3' \\
\hline qnrA & F: 5' - ATTTCTCACGCCAGGATTTG - 3R: 5' - GATCGGCAAAGGTTAGGTCA - 3' \\
\hline
\end{tabular}

a Abbreviations: TEM, Temineira; CTX-M, cefotaxime-Munich; SHV, Sulphydryl variable; qnrA, plasmid mediated Quinolone resistance determinants.

\begin{tabular}{lccc}
\hline Table 2. Polymerase Chain Reaction Programs Used in This Study & & \\
\hline bla SHV Gene & bla TEM Gene & bla CTX-M Gene & qnrA Gene \\
\hline $\begin{array}{l}3 \text { Minutes } 94^{\circ} \mathrm{C} \text { initial denatur- } \\
\text { ation }\end{array}$ & $\begin{array}{c}3 \text { Minutes } 94^{\circ} \mathrm{C} \text { initial denatur- } \\
\text { ation }\end{array}$ & $\begin{array}{c}3 \text { Minutes } 94^{\circ} \mathrm{C} \text { initial denatur- } \\
\text { ation }\end{array}$ & $\begin{array}{c}3 \text { Minutes } 94^{\circ} \mathrm{C} \text { initial denatur- } \\
\text { ation }\end{array}$ \\
1 Minute $94^{\circ} \mathrm{C}$ denaturation & 1 Minute $94^{\circ} \mathrm{C}$ denaturation & 1 Minute $94^{\circ} \mathrm{C}$ denaturation & 1 Minute $94^{\circ} \mathrm{C}$ denaturation \\
30 Seconds $58^{\circ} \mathrm{C}$ annealing & 53 Seconds $30^{\circ} \mathrm{C}$ annealing & 1 Minute $55.5^{\circ} \mathrm{C}$ annealing & 1 Minute $55^{\circ} \mathrm{C}$ annealing \\
1 Minute $72^{\circ} \mathrm{C}$ extension & 1 Minute $72^{\circ} \mathrm{C}$ extension & 1 Minute $72^{\circ} \mathrm{C}$ extension & 1 Minute $72^{\circ} \mathrm{C}$ extension \\
10 Minutes $72^{\circ} \mathrm{C}$ final extension & 5 Minutes $72^{\circ} \mathrm{C}$ final extension & 10 Minutes $72^{\circ} \mathrm{C}$ final extension & 10 Minutes $72^{\circ} \mathrm{C}$ final extension \\
30 Cycles & 30 Cycles & 30 Cycles & 30 Cycles \\
\hline
\end{tabular}

\footnotetext{
a Abbreviations: bla CTX-M, cefotaxime-Munich; bla SHV, sulphydryl variable; bla TEM, Temoneira; qnrA, plasmid mediated Quinolone resistance determinants.
} 
Abdi S et al.

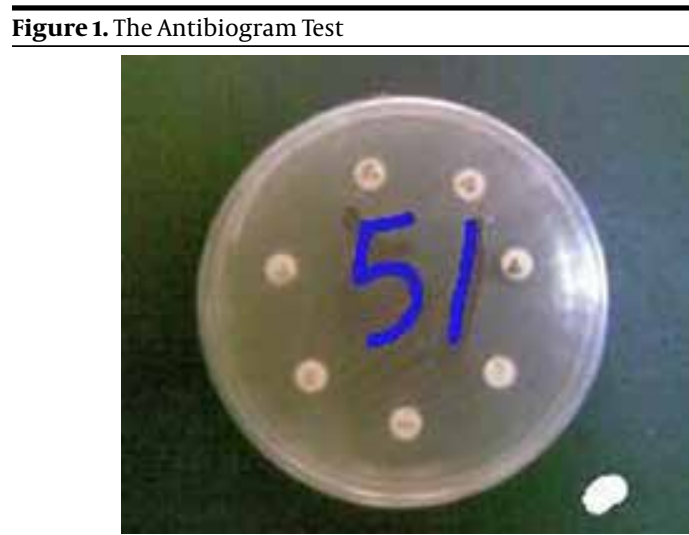

This Escherichia coli isolate was resistant to all tested antibiotics.

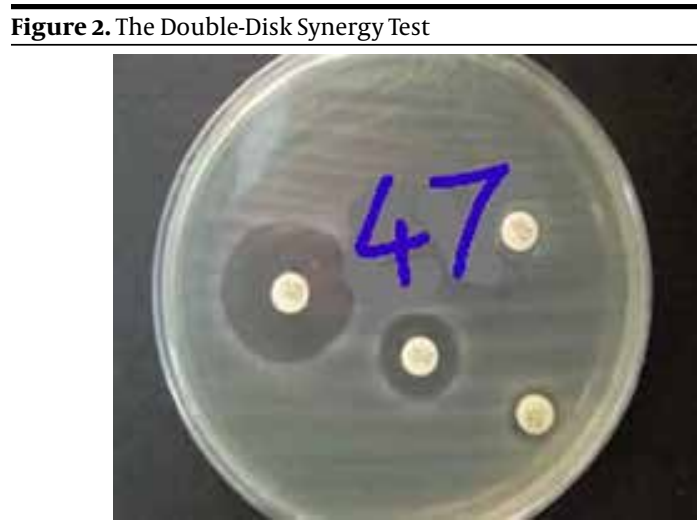

Increase of $\geq 5 \mathrm{~mm}$ in the growth inhibition zone around the disk containing cefotaxime with clavulanic acid versus cefotaxime for Escherichia coli. No 47 showed the extended-spectrum beta-lactamases-positive strains in this case.

Figure 3. The Frequency of blaCTX-M, bla TEM, bla SHV, and qnrA Genes Among Escherichia coli Isolates

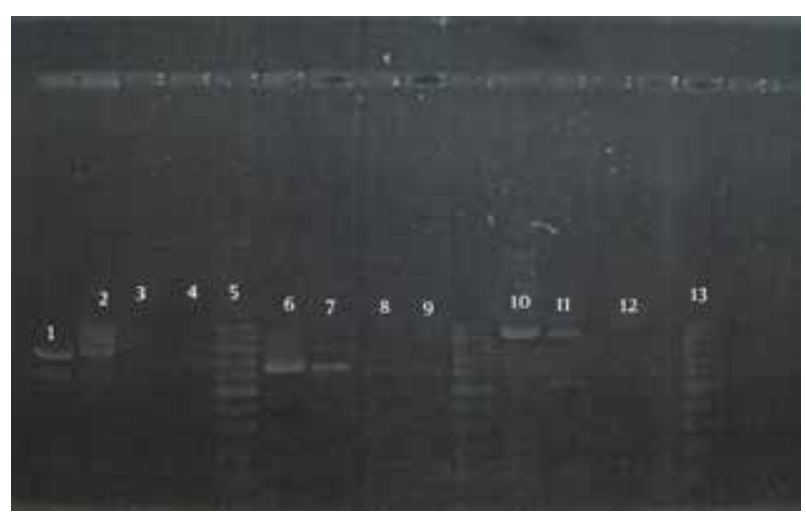

Lane 1, the 857 bp PCR product is related to bla CTX-M gene; lane 2, positive control (Klebsiella pneumonia ATCC 700603); lane 3, negative control (Escherichia coli ATCC 25922); lane 4, negative sample; lane 5, ladder (1001000) Fermentas, UK; lane 6, The 615 bp PCR product of bla SHV gene; lane 7, positive control (K. pneumonia ATCC 700603); lane 8, negative control (E. coli ATCC 25922); lane 9, ladder (100-1000) Fermentas, UK; lane 10, the 1150 bp bla TEM gene in clinical samples; lane 11, positive control (K. pneumonia ATCC 700603); lane 12, negative control; and lane 13, ladder (100-1000) Fermentas, UK.

\section{Figure 4. The Frequency of qnrA Gene by Polymerase Chain Reaction}

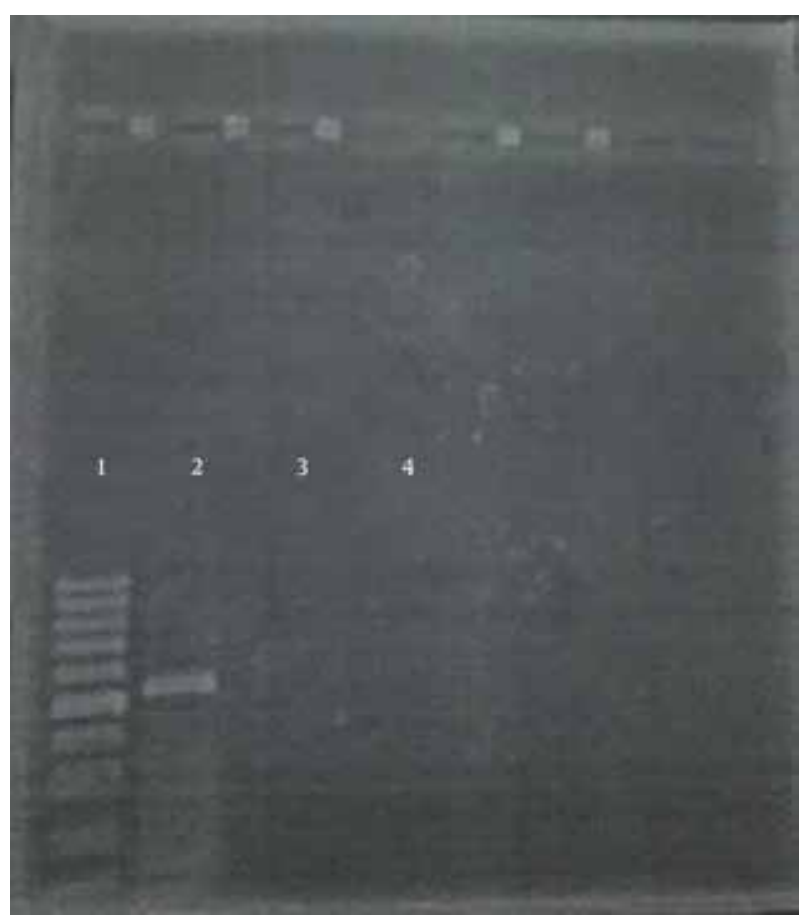

Lane 1, ladder (100-1000) Fermentas, UK; lane 2, the $516 \mathrm{bp} \mathrm{PCR} \mathrm{product} \mathrm{of}$ qnrA; lane 3, negative control (Escherichia coli ATCC 25922); and lane 4, a clinically negative case.

\section{Discussion}

In this study, the rate of resistance of the isolated E. coli strains to ampicillin and penicillin was $100 \%$ and this is an alarm for clinicians to pay more attention to the amount of consumption and prescription of these antibiotics. From 2002 to 2004, in Minnesota, USA, 931 specimens of $E$. coli were isolated from the urine samples of two groups, i.e. patients and controls, and their resistance to the fluoroquinolones was studied. In addition, the existence of resistance genes to fluoroquinolones, trimethoprim/sulfamethoxazole, and cephalosporins as well as the ability of ESBL production were analyzed by molecular PCR method. According to the results, the resistance rate to fluoroquinolones was higher in the hospitalized patients than in controls (15). Resistance of these bacteria was 100\% to penicillin and amoxicillin, $43 \%$ to cephalexin, $46 \%$ to ceftriaxone, and $2 \%$ to imipenem; these results revealed a high trend of resistance to the common antibiotics used in the routine treatment of UTIs. In addition, Warburg et al. studied the resistance rate of E. coli isolates to fluoroquinolones and screened the frequency of qnrA, qnrB and $a a c\left(6^{\prime}\right)-l b$-cr genes by using PCR during 1991-2005; they found that not only is the resistance to ciprofloxacin growing but also most of these strains produce ESBL. Therefore, gene translocation by plasmids plays an important role in this regard. Moreover, taking the results into account, a hypothesis suggests that there 
is a correlation between resistance to fluoroquinolones and synchronous ability of ESBL production because of the relation between these two plasmids (16). For this reason, the existence of qnrA gene among ESBL-positive $E$. coli strains was evaluated and based on its results, special attention must be paid to these strains during therapy.

In a five-year study in Houston, Texas, USA, the relation between resistance to fluoroquinolones and age as well as sex was studied and it revealed that the increase in age and duration of hospitalization accelerated the rate of resistance to fluoroquinolones in males (17). In another study in Norway, resistance of E. coli strains, taken from the urine of the patients with UTI, to ciprofloxacin and nalidixic acid by disk diffusion method showed 1\% increase during a four-year period. In the mentioned study, most specimens were taken from women (83\%). Then resistance to ciprofloxacin, trimethoprim, ampicillin, sulfonamide, nitrofurantoin, nalidixic acid, chloramphenicol, tetracycline, and gentamicin was studied by the phenotypic method and finally by using PCR and pulse-field method and the presence of genes involved in resistance was determined (18). Resistance to two routine drugs of the fluoroquinolones group, namely, nalidixic acid and ciprofloxacin was determined respectively to be $14 \%$ and $2 \%$ by using the disk diffusion method. In our study, resistance rate to nalidixic acid and ciprofloxacin were $53 \%$ and $51 \%$, respectively. In a study by Johnson et al. in Canada, the changes in resistance to ofloxacin, a fluoroquinolone, were evaluated; they found that the resistance rate to this drug had increased from $1 \%$ to $9 \%$ within a five-year period. They concluded that factors like duration of hospitalization and irregular use of these drugs had led to increased resistance (19). Similarly, increase in resistance of $E$. coli isolated from the urine of patients with UTIs to ciprofloxacin was attributed to the irregular consumption of fluoroquinolones in Switzerland (20).

According to the results of our study, the rate of resistance to beta-lactam drugs is increasing and treatment is difficult. This increasing trend of resistance has been seen with fluoroquinolones and synchronous resistance to these two antibiotic groups has been seen in most strains. As a result, we recommend to perform antibiogram test before prescribing any drug in order to prevent the increasing trend of resistance to fluoroquinolones and beta-lactam drugs.

\section{Acknowledgements}

We would like to express our thanks to the personnel of Molecular Biology Research Center, Baqiyatallah University of Medical Sciences, Tehran, Iran, for their help in conducting this project.

\section{Authors' Contributions}

The study concept and design was done by Mojdeh Hakemi Vala and Reza Ranjbar. Analysis and interpretation was done by Shima Abdi and Mojdeh Hakemi Vala.
Administration of technical and material supports was done by Shima Abdi, Ozra Baghery Bejestani, and Fatemeh Baghery Bejestany. Mojdeh Hakemi vala and Reza Ranjbar supervised the study. Statistical analysis was done by Shima Abdi. Drafting of the manuscript, critical revision of the manuscript, and intellectual contents was done by Mojdeh Hakemi Vala.

\section{Funding/Support}

This study was supported by grant of Baqiyatallah University of Medical Sciences, Tehran, Iran.

\section{References}

1. Gallini A, Degris E, Desplas M, Bourrel R, Archambaud M, Montastruc JL, et al. Influence of fluoroquinolone consumption in inpatients and outpatients on ciprofloxacin-resistant Escherichia col in a university hospital. J Antimicrob Chemother. 2010;65(12):2650-7.

2. Lee $\mathrm{K}$, Lee MA, Lee $\mathrm{CH}$, Lee J, Roh $\mathrm{KH}$, Kim S, et al. Increase of ceftazidime- and fluoroquinolone-resistant Klebsiella pneumoniae and imipenem-resistant Acinetobacter spp. in Korea analysis of KONSAR study data from 2005 and 2007. Yonsei Med J. 2010;51(6):901-11.

3. Azap OK, Arslan $\mathrm{H}$, Serefhanoglu K, Colakoglu S, Erdogan $\mathrm{H}$ Timurkaynak F, et al. Risk factors for extended-spectrum betalactamase positivity in uropathogenic Escherichia coli isolated from community-acquired urinary tract infections. Clin Microbiol Infect. 2010;16(2):147-51.

4. Ehlers MM, Veldsman C, Makgotlho EP, Dove MG, Hoosen AA, Kock MM. Detection of blaSHV, blaTEM and blaCTX-M antibiotic resistance genes in randomly selected bacterial pathogens from the Steve Biko Academic Hospital. FEMS Immunol Med Microbiol. 2009;56(3):191-6.

5. Paterson DL, Bonomo RA. Extended-spectrum beta-lactamases: a clinical update. Clin Microbiol Rev. 2005;18(4):657-86.

6. Lee JH, Bae IK, Lee SH. New definitions of extended-spectrum beta-lactamase conferring worldwide emerging antibiotic resistance. Med Res Rev. 2012;32(1):216-32.

7. Jacoby GA, Sutton L. Properties of plasmids responsible for production of extended-spectrum beta-lactamases. Antimicrob Agents Chemother. 1991;35(1):164-9.

8. Gales AC, Jones RN, Turnidge J, Rennie R, Ramphal R. Characterization of Pseudomonas aeruginosa isolates: occurrence rates, antimicrobial susceptibility patterns, and molecular typing in the global SENTRY Antimicrobial Surveillance Program, 19971999. Clin Infect Dis. 2001;32 Suppl 2:S146-55.

9. Murray PR, Jorgenson JH, Yolken RH, Baron EJ, Pfaller MA. Manual of clinical microbiology. 8 th ed: Mosby; 2003.

10. Performance standards for Antimicrobial Susceptibility Testing Twenty-second Informational Supplement.USA: Clinical and Laboratory Standards Institute; 2010.

11. Livermore DM, Hope R, Brick G, Lillie M, Reynolds R, Bsac Working Parties on Resistance Surveillance . Non-susceptibility trends among Enterobacteriaceae from bacteraemias in the UK and Ireland, 2001-06. J Antimicrob Chemother. 2008;62 Suppl 2:ii41-54.

12. Lee S, Park YJ, Kim M, Lee HK, Han K, Kang CS, et al. Prevalence of Ambler class A and D beta-lactamases among clinical isolates of Pseudomonas aeruginosa in Korea. J Antimicrob Chemother 2005;56(1):122-7.

13. Feizabadi MM, Delfani S, Raji N, Majnooni A, Aligholi M, Shahcheraghi F, et al. Distribution of bla(TEM), bla(SHV), bla(CTX-M) genes among clinical isolates of Klebsiella pneumoniae at Labbafinejad Hospital, Tehran, Iran. Microb Drug Resist. 2010;16(1):49-53.

14. Vila J, Ruiz J, Marco F, Barcelo A, Goni P, Giralt E, et al. Association between double mutation in gyrA gene of ciprofloxacin-resistant clinical isolates of Escherichia coli and MICs. Antimicrob Agents Chemother. 1994;38(10):2477-9.

15. Johnson JR, Sannes MR, Croy C, Johnston B, Clabots C, Kuskowski 
MA, et al. Antimicrobial drug-resistant Escherichia coli from humans and poultry products, Minnesota and Wisconsin, 20022004. Emerg Infect Dis. 2007;13(6):838-46.

16. Warburg G, Korem M, Robicsek A, Engelstein D, Moses AE, Block C, et al. Changes in aac ( $\left.6^{\prime}\right)$-Ib-cr prevalence and fluoroquinolone resistance in nosocomial isolates of Escherichia coli collected from 1991 through 2005. Antimicrob Agents Chemother. 2009;53(3):1268-70

17. Boyd LB, Atmar RL, Randall GL, Hamill RJ, Steffen D, Zechiedrich L. Increased fluoroquinolone resistance with time in Escherichia coli from $>17,000$ patients at a large county hospital as a function of culture site, age, sex, and location. BMC Infect Dis. 2008;8:4.
18. Grude N, Strand L, Mykland H, Nowrouzian FL, Nyhus J, Jenkins A, et al. Fluoroquinolone-resistant uropathogenic Escherichia coli in Norway: evidence of clonal spread. Clin Microbiol Infect. 2008;14(5):498-500.

19. Johnson L, Sabel A, Burman WJ, Everhart RM, Rome M, MacKenzie TD, et al. Emergence of fluoroquinolone resistance in outpatient urinary Escherichia coli isolates. Am J Med. 2008;121(10):876-84.

20. Nicoletti J, Kuster SP, Sulser T, Zbinden R, Ruef C, Ledergerber B, et al. Risk factors for urinary tract infections due to ciprofloxacinresistant Escherichia coli in a tertiary care urology department in Switzerland. Swiss Med Wkly. 2010;140:w13059. 